\title{
INHIBICIÓN DE LA OXIDACIÓN IN VITRO DE LIPOPROTEÍNAS DE BAJA DENSIDAD (LDL), POR EXTRACTOS ACUOSOS DE CAMELLIA SINENSIS E HIBISCUS SABDARIFFA
}

\author{
Inhibition of low-density lipoprotein (LDL) oxidation in vitro by aqueous \\ extracts of Camellia
}

\section{Franklin Jesús Pacheco Coello ${ }^{a}$, Doralys Alessandra Ramírez Azuaje ${ }^{b}$, Corymar Pastora Orosco Vargas ${ }^{c}$, María Milagro Peraza Marrero ${ }^{d}$ y Ibis Coromoto Pinto Catari ${ }^{b}$}

Recibido: 10 de abril, $2020 \bullet$ Aprobado: 5 de mayo, 2020

Cómo citar: Pacheco Coello FJ, Ramírez Azuaje DA, Orosco Vargas CP, Peraza Marrero MM, Coromoto Pinto Catari I. Inhibición de la oxidación in vitro de lipoproteínas de baja densidad (LDL), por extractos acuosos de Camellia sinensis e Hibiscus sabdariffa. cysa [Internet]. 18 de septiembre de 2020 [citado 18 de septiembre de 2020];4(3):5-11. Disponible en: https://revistas.intec.edu.do/index. php/cisa/article/view/1924

\section{Resumen}

Introducción: el uso de bebidas funcionales como las infusiones de Camellia sinensis e Hibiscus sabdariffa, ha crecido en los últimos ańos a nivel mundial gracias a su contenido en compuestos bioactivos con un alto poder antioxidante, atribuyéndoles diversos efectos entre los que destaca su capacidad hipolipemiente.

Objetivo: el estudio tuvo como propósito evaluar la capacidad de dos extractos acuosos de $C$. sinensis e $H$. sabdariffa en la inhibición de la oxidación de las LDL obtenidas de plasma.

Material y métodos: se emplearon hojas de té verde orgánico y cálices deshidratados para la obtención de los extracto. Se determino el contenido de fenoles totales por el método de Folin-Ciocalteu y el ensayo de la oxidación de las LDL como modelo biológico para la evaluación de la capacidad inhibitoria de los extractos.

\footnotetext{
a Docente-Investigador, Universidad de Carabobo, Venezuela. BIOMED-UC. ORCID: 0000-0002-2765-4069

b ORCID: 0000-0002-4947-8027

Correo-e: doralysramirez@gmail.com

c ORCID: 0000-0002-3173-1004

Correo-e: cpov04@gmail.com
}

\begin{abstract}
Introduction: the use of functional beverages such as the infusions of Camellia sinensis and Hibiscus sabdariffa, has grown in recent years worldwide thanks to its content in bioactive compounds with a high antioxidant power, attributing various effects among which its lipid-lowering capacity stands out.
\end{abstract}

Objective: the purpose of the study was to evaluate the capacity of two aqueous extracts of $C$. sinensis and $H$. sabdariffa in inhibiting the oxidation of LDL obtained from plasma.

Material and methods: organic green tea leaves and dehydrated calyces were used to obtain the extracts. The total phenol content was determined by the Folin-Ciocalteu method and the LDL oxidation test as a biological model for the evaluation of the inhibitory capacity of the extracts.

\footnotetext{
${ }^{d}$ ORCID:0000-0001-5248-9854

Correo-e: mmm1504peraza@gmail.com

'ORCID:0000-0002-9333-9147

Correo-e: ibispintoc@gmail.com
} 
Resultados: se observó inhibición de la oxidación de las LDL por ambos extractos con diferencia estadística respecto al control $(p \leq 0,05)$, siendo el extractos de $C$. sinensis el mayor actividad.

Conclusiones: las dos especies aportan un alto contenido de antioxidantes fundamentales para el organismo, por lo que la ingesta regulada y dirigida por expertos podría contribuir sin duda al tratamiento de hiperlipidemia.

Palabra clave: Camellia sinensis; Hibiscus sabdariffa flavonoides; antioxidantes; hiperlipidemia

\section{Introducción}

El té verde (Camellia sinensis) y la flor conocida como rosa de jamaica (Hibiscus sabdariffa), están considerados entre las tres primeras bebidas de mayor consumo después del agua, y su popularidad ha crecido por sus diversas potencialidades y efectos a la salud ${ }^{1,2}$. Entre las propiedades que se les atribuyen están sus efectos antiinflamatorio, antimicrobiano, anticancerígeno, antioxidante, hipoglicemiante $y$, en especial, su capacidad de hipolipemiante ${ }^{3-5}$. Las actividades bilógicas anteriormente señaladas son gracias a su alto contenido en compuestos bioactivos como los polifenoles, que son un grupo cercano a 8.000 sustancias y que pueden ser clasificados de acuerdo con su estructura. Entre los más importantes están los flavonoides, que poseen una estructura básica C6-C3-C6, las antocianinas, catequinas y epicatequinas, estas últimas predominantes en C.sinensis ${ }^{6-8}$.

Con relación a la lipoproteína de baja densidad (LDL), se sabe que esta es avalada por estudios epidemiológicos y de intervención como mejor predictor de la enfermedad cardiovascular y coronaria que el colesterol total ${ }^{9,10}$. Sobre estas evidencias se han
Results: inhibition of LDL oxidation by both extracts was observed with statistical difference from the control $(\mathrm{p} \leq 0.05)$, with $C$. sinensis extracts being the highest activity.

Conclusions: the two species provide a high content of fundamental antioxidants for the body, so that regulated and expertly directed intake could certainly contribute to the treatment of hyperlipidemia.

Keywords: Camellia sinensis; Hibiscus sabdariffa flavonoids; antioxidants; hyperlipidemia.

apoyado las guías de práctica clínica, donde se considera el LDL como el objetivo terapéutico principal, y se establece en función del nivel de riesgo del paciente, un nivel objetivo definido ${ }^{11}$.

Todo lo expuesto anteriormente y considerando que en Venezuela hasta la fecha no hay reportes de estudios similares, se propuso evaluar el efecto inhibitorio de estos extractos en LDL obtenidas de plasmas de pacientes con hiperlipidemia.

\section{Material y métodos}

\section{Material vegetal}

Se emplearon hojas de té verde orgánico procedente de China los cuales permanecieron en su envase original hasta su procesamiento. Por otra parte, los cálices de $H$. sabdariffa, fueron obtenidos de plantaciones propias por los investigadores, en el estado Aragua, Venezuela, entre junio y diciembre de 2019. Estos fueron deshidratados y almacenados libre de humedad, hasta su análisis en el Laboratorio de Metales Pesados y Solventes Orgánicos de la Universidad de Carabobo, sede Aragua (figuras 1 y 2). 

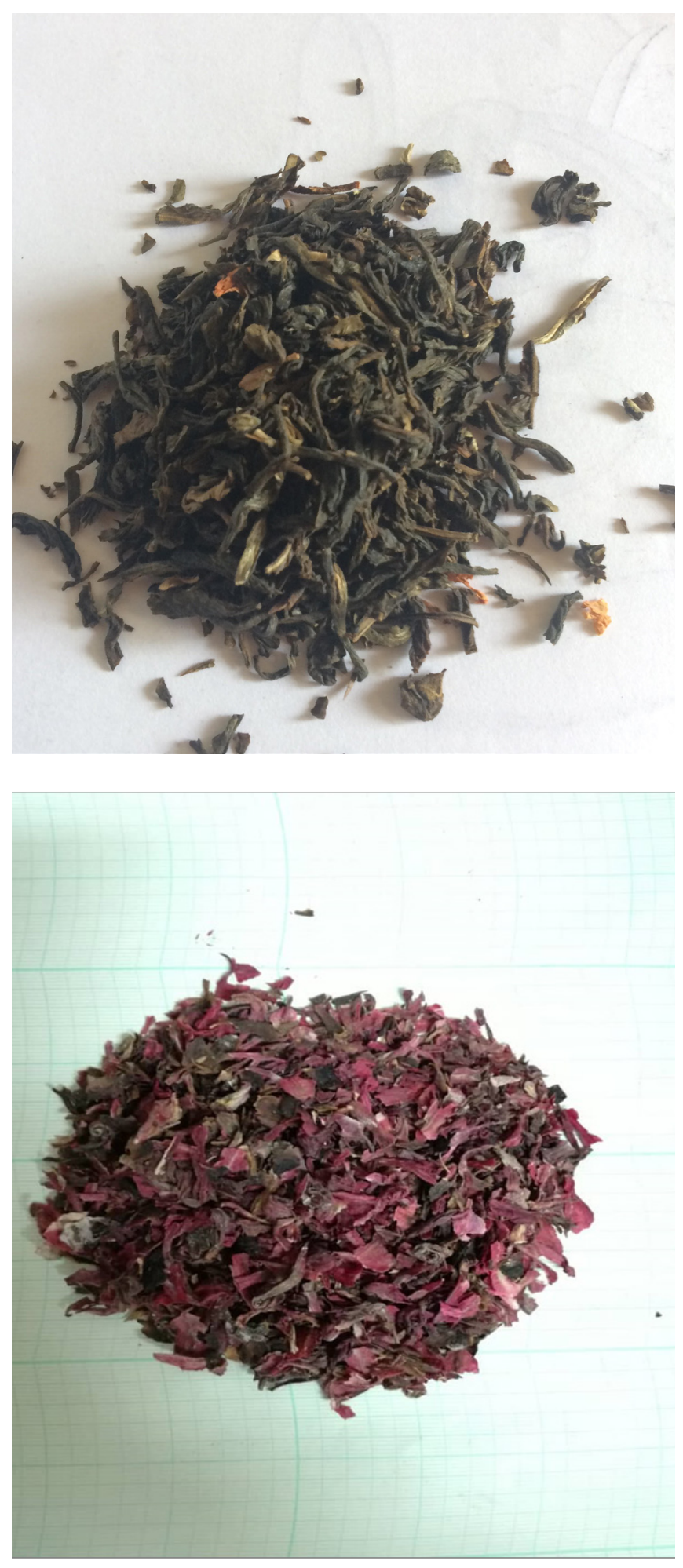

Figuras 1 y 2: hojas de té verde orgánico de $C$. sinensis y cálices deshidratados de $H$. sabdariffa

Fuente: elaboración propia.

\section{Obtención de los extractos acuosos}

Los extractos se prepararon con 2,5 g de material vegetal y $100 \mathrm{~mL}$ de agua destilada. Se dejó hervir por 15 minutos, se separó el líquido del resto de material vegetal por decantación y la extracción se repitió en las mismas condiciones por triplicado. Los extractos se filtraron con papel Whatman No. 4 y se aforó a $200 \mathrm{~mL}$ con agua destilada ${ }^{12}$.

\section{Obtención de las lipoproteínas de baja densidad en plasma $(\mathrm{LDL})$}

Se realizó la extracción sanguínea, previa asepsia y empleando un tubo de tapa morada con ácido etilendiaminotetraacético (EDTA) sellado al vacío con aguja vacutainer. Estas muestras fueron obtenidas de cinco pacientes de los cuales uno de ellos no presentaba hiperlipidemia (todos en ayuna de 8 horas). A cada paciente se le explicó el propósito de la investigación, obteniendo así su consentimiento informado, siguiendo los lineamientos de la Declaración de Helsinki y del Consejo Técnico del Centro de Estudio en Salud de los Trabajadores de la Universidad de Carabobo.

\section{Determinación de fenoles totales}

La determinación de fenoles se realizó por el método colorimétrico de Folin-Ciocalteu. $50 \mu \mathrm{L}$ de muestra fueron adicionados a $125 \mu \mathrm{L}$ del reactivo de Folin, y $400 \mu \mathrm{L}$ de carbonato de sodio 7,1 \% (p/v), completándose con agua destilada hasta $1 \mathrm{~mL}$. Este procedimiento se realizó por quintuplicado. Seguidamente se prepararon 5 patrones de concentración de 50,100, 150, 200 y $250 \mu \mathrm{g} / \mathrm{mL}$, a partir de una solución patrón madre de Ácido Gálico (fenol) de concentración $500 \mu \mathrm{g} / \mathrm{mL}$. Por último, se realizó la lectura a $760 \mathrm{~nm}$ empleando el equipo de absorción molecular Génesis 20 (Thermo Scintific). Los resultados se expresaron como mg de GAE / g de material vegetal $(\mathrm{MV})^{13}$. 


\section{Ensayo de oxidación de LDL}

Los tubos se agitaron con un vórtex y llevados a una temperatura de $2{ }^{\circ} \mathrm{C}$ durante $15 \mathrm{~min}$, para luego ser nuevamente agitados y centrifugados a $5000 \mathrm{rpm}$ a $4{ }^{\circ} \mathrm{C}$ durante $5 \mathrm{~min}$. El sobrenadante que corresponde al plasma se transfirió volumétricamente a un tubo falcon estéril de $15 \mathrm{~mL}$ y se adicionó el reactivo precipitante (ácido fosfotúngstico $500 \mathrm{mM}$ y cloruro de magnesio $500 \mathrm{mM}$ ) en relación 2:1 $v / v$ con el plasma obtenido. El reactivo precipitante y el plasma se agitaron durante 2 min en vórtex y, posteriormente, se centrifugaron a $3000 \mathrm{rpm} \mathrm{a} 4{ }^{\circ} \mathrm{C}$ durante 10 min. El sobrenadante obtenido (LDL) este se con buffer de fosfatos $10 \mathrm{mM}$ y $0,16 \mathrm{M}$ de $\mathrm{NaCl}, \mathrm{pH}$ 7,4 hasta $100 \mathrm{~mL}$. Seguidamente se llevó a cabo la oxidación de las LDL que consistió en tomar $115 \mu \mathrm{L}$ de solución de LDL, $100 \mu \mathrm{L}$ de extracto acuoso con buffer de fosfatos, $235 \mu \mathrm{L}$ de buffer de fosfatos salino y $50 \mu \mathrm{L}$ de $\mathrm{CuSO}_{4} 100 \mu \mathrm{M}$, el cual actúa como oxidante de las LDL. La mezcla se agitó en vórtex durante 2 min y luego se incubó a $37^{\circ} \mathrm{C}$ en agitación durante $8 \mathrm{~h}$. Para detener la oxidación las muestras fueron transferidas por una columna de Sephadex G-50, tomando $550 \mu \mathrm{L}$ del eluido y $500 \mu \mathrm{L}$ de ácido tricloroacético (ATA) 25 $\% p / v$. Posteriormente, se adicionaron $500 \mu \mathrm{L}$ de ácido tiobarbitúrico $1 \% p / v$. Se agitó nuevamente en vórtex durante $1 \mathrm{~min}$ y se incubó a $95^{\circ} \mathrm{C}$ por 1 $\mathrm{h}$ en oscuridad. Luego, se dejó enfriar durante $1 \mathrm{~h}$, en oscuridad a temperatura ambiente $\left(25^{\circ} \mathrm{C}\right)$ y se centrifugó a $5000 \mathrm{rpm}$ durante $5 \mathrm{~min}$. Se realizó una curva de calibración del método de sustancias reactivas al ácido tiobarbitúrico (TBARS) y se utilizó como estándar 1,1,3,3-tetrametoxipropano (TMP) en concentraciones de 0,5 a $10 \mu \mathrm{M}$. Se midieron las absorbancias de las muestra, patrones, control (paciente sin hiperlipidemia) y los resultados se expresaron como $\mu \mathrm{g}$ TMP/100 g de MV ${ }^{14,15}$.

\section{Análisis estadístico}

Todas las determinaciones de las muestras y control se realizaron por triplicado. Para comparar cada efecto del extracto respecto a su control de cada paciente, se aplicó un análisis de varianza de dos vías con interacción (ANOVA), usando el programa Statistix 9.0 para Windows.

\section{Resultados}

La determinación de fenoles totales arrojó concentraciones de 150,03 $\pm 2,23 \mathrm{mg}$ de GAE/g de MV para $C$. sinensis y $14,12 \pm 1,03 \mathrm{mg}$ de GAE/g de MV para $H$. sabdariffa. La evaluación de los extractos en la inhibición de la oxidación de las LDL, evidenció que para cada uno de los ensayos la oxidación fue estadísticamente significativo $(p \leq 0,05)$ al comprarse con el control, siendo el extracto de $C$. sinensis donde más se observó el efecto inhibitorio (figuras 3 y 4).

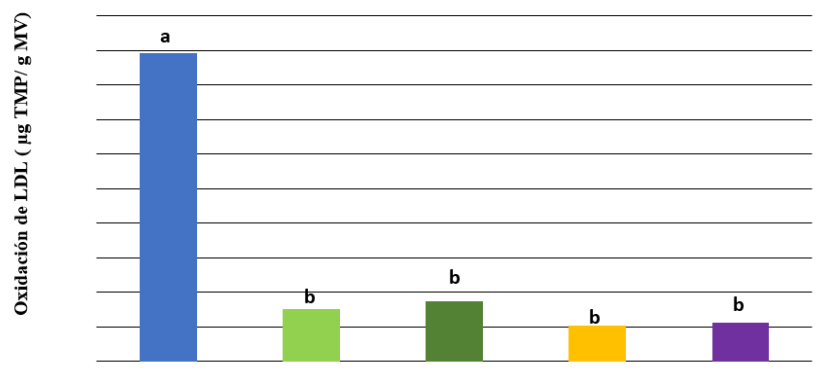

Figura 3. Inhibición de la oxidación de las LDL por el extracto acuoso de Camellia sinensis. $\mathrm{Mp}=$ muestra paciente, Control (paciente sin hiperlipidemia), a y b estadísticamente significativo $(p \leq 0,05)$

Fuente: elaboración propia.

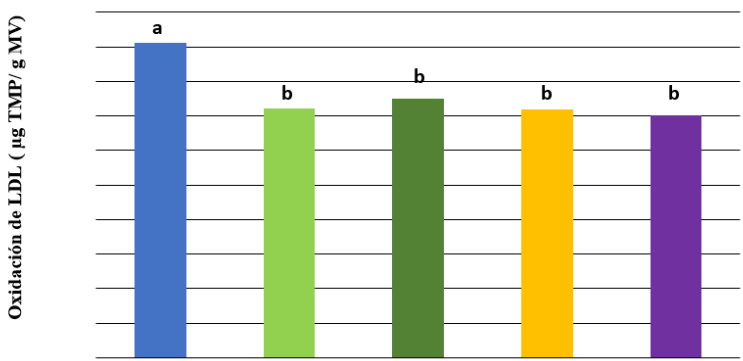

Figura 4. Inhibición de la oxidación de las LDL por el extracto acuoso de Hibiscus sabdariffa. Mp=muestra paciente, Control (paciente sin hiperlipidemia), a y b estadísticamente significativo $(p \leq 0,05)$

Fuente: elaboración propia. 


\section{Discusión}

El efecto observado en los ensayos, evidencian que los compuestos bioactivos presentes en $C$. sinensis e $H$. sabdariffa, poseen una capacidad antioxidante semejante a lo hallado en diversos estudios y superior a lo reportado en extractos de frutas tropicales como curuba (Passiflora tripartida var. Mollissima), gulupa (Passiflora edulis Sims), aguacate variedad Hass (Persea America Mill), lulo (Solanum quitoense Lam.), tomate de árbol (Cyphomandra betacea Sendt) y uchuva (Physalis peruviana L) ${ }^{16,17}$. Es importante señalar que la actividad antioxidante observada en ambos extractos está en relación con la concentracion de los compuestos fenólicos presentes en el material vegetal, los cuales varían de una cosecha a otra debido factores climaticos, calidad del suelo, uso de fertilizantes, genética de la planta entre otros. ${ }^{18}$

En este sentido, se evidenció una mayor inhibición de la oxidación de las LDL, con el extracto de té verde orgánico que con el extracto de H.sabdariffa, en cada una de las muestras de los pacientes con hiperlipidemia, esto debido a su alto contenido en antioxidantes, entre los que destacan: epicatequina, galato de epicatequina, epigalocatequina y galato de epigalocatequina, a diferencia de de H.sabdariffa donde el responsable de dicha acción son las antocianinas ${ }^{2,19}$. Si bien otros estudio han evaluado la capacidad de reducir los niveles de LDL, colesterol total y triglicéridos por medio de ingesta regulada de infusiones de $C$. sinensis e $H$. sabdariffa, todos concluyen que la biodisponibilidad (ingesta), el metabolismo y factores genéticos (polimorfismo de enzimas) son fundamentales, de ahí la importancia de evaluarlos de la mano al tratamiento, para obtener los efectos benéficos a la salud en pro de evitar problemas cardiovasculares a causa de la oxidación de estas lipoproteínas ${ }^{20-23}$.

\section{Conclusiones}

El uso de infusiones a base de hojas de té verde y cálices de $H$. sabdariffa, son una alternativa indiscutible en el consumo de compuestos bioactivos.
A pesar que el efecto esperado in vivo a lo hallado en in vitro, no suele ser tan fácil por la biodisponibildad de estos antioxidantes en la dieta diaria, el control y la supervisión por expertos en el consumo de estas infusiones puede traer una mejoría significativa al ser humano y reducir eventos cardiovasculares.

\section{Bibliografía}

1. Hashimoto T, Goto M, Sakakibara H, Oi N, Okamoto M, Kanazawa K. Yellow tea is more potent than other types of tea in suppressing liver toxicity induced by carbon tetrachloride in rats. Phytother Res. 2007;2:668-70

2. Ghazala R, Rajni C. A review on phytochemistry and therapeutic uses of Hibiscus sabdariffa $\mathrm{L}$. Biomedicine Pharmacothe. 2018;102(1):575-6.

3. Quideau S, Deffieux D, Douat-Casassus C, Pouységu L. Plant polyphenols: chemical properties biological activities, and synthesis. Angew Chem Int. 2011;50:586-621

4. Ninfali P, Mea G, Giorgini S, Rocchi M, Bacchiocca M. Antioxidant capacity of vegetables, spices and dressings relevant to nutrition. Br J Nutr. 2005;93:257-66.

5. Tang J, Zheng JS, Fang L, Jin Y, Cai W, Li D. Tea consumption and mortality of all cancers, CVD and all causes: a meta-analysis of eighteen prospective cohort studies. Br J Nutr. 2015;114:673-83.

6. Gaviria C, Ochoa C, Sánchez N, Medina C, Lobo M, Galeano P. Actividad antioxidante e inhibición de la peroxidación lipídica de extractos de frutos de mortiño (Vaccinium meridionale Sw). Blacpma. 2005;8(1):519-28.

7. Choksi RB, Boylston WH, Rabek WR, Widger JP. Oxidatively damaged proteins of heart mitochondrial elect ron transport complexes. Biochim Biophys Acta. 2004; 168:95-101 
8. Rojano B, Zapata, K, Cortés F. Capacidad atrapadora de radicales libres de Passiflora mollissima (Kunth) L.H. Bailey (curuba), Rev Cubana Plantas Med. 2012;17:408-19.

9. Van Deventer HE, Greg Miller W, Myers GL, Sakurabayashi I, Bachmann LM, Caudill SP et al. El colesterol no-HDL demuestra una mejor exactitud en el score de la clasificación del riesgo cardiovascular comparado con el colesterol LDL, directo o calculado, en una población dislipémica. Acta Bioquim Clin Latinoam. 2011;45(4):773-84.

10. Armas Rojas NB, de la Noval García R, Dueñas Herrera A, Castillo Nuñez JC, Suárez Medina R, Castillo Guzmán A. Estimación del riesgo cardiovascular mediante tablas de la Organización Mundial de la Salud. Área de salud "Héroes del Moncada". Rev Cubana Cardiol Cir Cardiovasc. 2014;20(1):34-55.

11. Argüeso Armesto R, Díaz Díaz JL, Díaz Peromingo JA, Rodríguez González A, Castro Mao M, Diz Lois F. Lípidos, colesterol y lipoproteínas. Galicia Clin. 2011;72(1):23-65

12. Reyes-Luengas A, Salinas-Moreno Y, Ovando-Cruz M, Arteaga-Garibay R. Análisis de ácidos fenólicos y actividad antioxidante de extractos acuosos de variedades de jamaica (Hibiscus Sabdariffa L.) con cálices de colores diversos. Agrociencia. 2015;49:277-90

13. Singleton VL, Rossi JA. Colorimetry of total phenolics with phosphomolybdic-phosphotungstic acid reagents. Am J Enol Vitic. 1965; 16(3):144-58.
14. Ruiz F, Giacopini M, Landaeta M. Bosch V. Susceptibilidad a la oxidación de las lipoproteínas de baja y muy baja densidad de plasma en escolares. Ana Vene Nutri. 2006;19(1):1-7.

15. Da-Costa-Rocha I, Bonnlaender B, Sievers H, Pischel I, Heinrich M. Hibiscus sabdariffa L. A phytochemical and pharmacological review. Food Chem. 2014;165:424-43.

16. Albarrán G, Mendoza E, Beltrán J. M. Influence of concentration on the radiolytic decomposition of thiamine, riboflavin, and pyridoxine in aqueous solution. Rev Colomb Quim. 2014;43(3):41-48.

17. Bresciani L, Calani L, Cossu M, Mena P, Sayegh M, Ray S. (Poly)phenolic characterization of three food suplements containing 36 different fruits, vegetable and berries. Pharma Nutrition. 2015;311-9.

18. Reyes-Luengas A, Salinas-Moreno Y, Ovando-Cruz M, Arteaga-Garibay R. Análisis de ácidos fenólicos y actividad antioxidante de extractos acuosos de variedades de jamaica (Hibiscus Sabdariffa L.) con cálices de colores diversos. Agrociencia. 2015;49:277-90

19. Serafini M, Del Rio D, Yao DN, Bettuzzi S, Peluso I . Chapter . Health benefits of tea. In: Benzie IFF, Wachtel-Galor S (2eds). Herbal Medicine: Biomolecular and Clinical Aspects, CRC Press/Taylor \& Francis:,USA: Boca Raton; 2011. p.12-13.

20. Huang TW, Chang CL, Kao ES, Lin JH. Effect of Hibiscus sabdariffa extract on high fat diet-induced obesity and liver damage in hamsters, Food Nutr. Res. 2015; 59:234-56. 
21. Lin HH, Charles AL, Hsieh CW, Lee YC, Ciou JY. Antioxidant effects of 14 Chinese traditional medicinal herbs against human low-density lipoprotein oxidation, J. Tradit. Complement. Med. 2015;5:51-5

22. Peluso I, Serafini M. Antioxidants from black and green tea: from dietary modulation of oxidative stress to pharmacological mechanisms. Br. J. Pharmacol. 2017;174:1195-208.
23. Zhao CN, Tang GY, Cao SY. Phenolic Profiles and Antioxidant Activities of 30 Tea Infusions from Green, Black, Oolong, White, Yellow and Dark Teas. Antioxidants. 2019;8(7):200-15. 